\title{
Estudo prospectivo sobre o impacto do uso do selante de fibrina humano livre de agentes estabilizadores de coágulo na artroplastia total de joelho*
}

\section{Prospective Study on the Impact of the Use of Human Fibrin Sealant free of Clot-Stabilizing Agents in Total Knee Arthroplasty}

\author{
Douglas Mello Pavão ${ }^{10}$ Guilherme Mathias Palhares ${ }^{1}$ Rodrigo Satamini Pires e Albuquerque ${ }^{1}$ \\ Eduardo Branco de Sousa ${ }^{1}$ João Maurício Barretto ${ }^{1}$ \\ ${ }^{1}$ Instituto Nacional de Traumatologia e Ortopedia Jamil Haddad, Rio \\ de Janeiro, RJ, Brasil \\ Rev Bras Ortop 2019;54:322-328. \\ Address for correspondence Douglas Mello Pavão, MD, MSc, Instituto \\ Nacional de Traumatologia e Ortopedia Jamil Haddad, Avenida Brasil, \\ 500, Rio de Janeiro, RJ, 20940-070, Brasil \\ (e-mail: drdouglaspavao@gmail.com).
}

Trabalho feito no Instituto Nacional de Traumatologia e Ortopedia Jamil Haddad, Rio de Janeiro, RJ, Brasil.

(1) Douglas Mello Pavão's ORCID is https://orcid.org/0000-0002-35320472.
Objetivo O objetivo do presente estudo foi avaliar os resultados do uso tópico intraoperatório do selante de fibrina humano livre de agentes estabilizadores de coágulo em pacientes com osteoartrite (OA) submetidos a artroplastia total de joelho (ATJ), buscando diferenças entre os grupos em relação à perda sanguínea, à necessidade transfusional, ao tempo de internação hospitalar, à percepção de dor, à amplitude de movimento e à incidência de complicações.

Métodos Foram analisados prospectivamente um grupo de intervenção (Selante) com 32 pacientes e um grupo controle (Controle) com 31 pacientes, com OA sintomática dos joelhos, submetidos a ATJ.

Resultados Os resultados foram semelhantes entre os grupos, em relação à perda sanguínea visível no dreno em 24 horas (Controle 276,5 mL $\pm 46,24$ versus Selante $365,9 \mathrm{~mL} \pm 45,73$ ), à perda sanguínea total em 24 horas (Controle $930 \mathrm{~mL} \pm 78$ versus Selante $890 \mathrm{~mL} \pm 67$ ) e em 60 horas de pós-operatório (Controle $1.250 \mathrm{~mL} \pm 120$ versus Selante $1.190 \mathrm{~mL} \pm 96$ ), à necessidade de hemotransfusão (ocorreu em apenas 1 controle), ao tempo de dias na permanência hospitalar (Controle 5,61 $\pm 0,50$ [ $n=31$ ] versus Selante $4,81 \pm 0,36$ ), dor pós-operatória e amplitude de movimento. O uso do agente selante de fibrina não se relacionou à ocorrência de complicações da cicatrização de ferida, de infecção ou de trombose venosa profunda.

Conclusão Concluímos que o agente hemostático de fibrina humana não foi eficaz em reduzir o volume de sangramento e a necessidade de hemotransfusão ou em interferir no tempo de internação hospitalar, na percepção de dor e na amplitude de movimento. Seu uso não se relacionou a nenhuma complicação. received

February 27, 2018

accepted

July 10,2018
DOI https://doi.org/

$10.1055 / \mathrm{s}-0039-1692447$.

ISSN $0102-3616$.
Copyright $(2019$ by Sociedade Brasileira License terms de Ortopedia e Traumatologia. Published by Thieme Revnter Publicações Ltda, Rio de Janeiro, Brazil 


\begin{abstract}
Keywords

- arthroplasty, replacement, knee

- osteoarthritis

- blood loss

- fibrin sealant

Objective The goal of the present study was to evaluate the results of the intraoperative topical use of a human fibrin sealant free of clot-stabilizing agents in total knee arthroplasties (TKAs), looking for differences between groups in relation to blood loss, transfusional need, length of hospital stay, pain perception, range of motion, and incidence of complications.

Methods We have analyzed prospectively an intervention group with 32 patients (Sealant) and a control group with 31 patients (Control) with symptomatic knee osteoarthritis who underwent total knee arthroplasty.

Results The results were similar between the groups, in relation to the visible blood loss in the drain in 24 hours (Control $276.5 \mathrm{~mL} \pm 46.24$ versus Sealant 365.9 $\mathrm{mL} \pm 45.73$ ), the total blood loss in 24 hours (Control $930 \mathrm{~mL} \pm 78$ versus Sealant $890 \mathrm{~mL} \pm 67$ ) and in the postoperative 60 hours (Control $1,250 \mathrm{~mL} \pm 120$ versus Sealant $1,190 \mathrm{~mL} \pm 96$ ), the need for blood transfusion (occurred in only 1 control), the length of hospital days stay (Control $5.61 \pm 0.50[n=31]$ versus Sealant $4.81 \pm 0.36$ ), postoperative pain and range of motion. Its use was not related to the occurrence of wound healing complications, of infection, or of deep venous thrombosis.

Conclusion We conclude that the hemostatic agent of human fibrin free of clotstabilizing agents was not effective in reducing bleeding volume and the need for blood transfusion or in interfering with hospital length of stay, pain perception and range of motion. Its use was not related with any complications.
\end{abstract}

\section{Introdução}

A osteoartrite $(\mathrm{OA})$ do joelho envolve a degeneração da cartilagem, inflamação sinovial e espessamento do osso subcondral, levando a dor e a limitação funcional. A artroplastia total do joelho (ATJ) é uma das opções de tratamento nos estágios finais da doença, reduzindo os sintomas e restaurando a função articular. O design e a fixação dos implantes têm melhorado progressivamente, levando a um aumento na sua sobrevida e função. No entanto, a perda sanguínea relacionada a este procedimento ainda é uma preocupação que precisa ser investigada e tratada. ${ }^{1-4}$

As estratégias de controle e de prevenção da perda sanguínea incluem o uso de torniquetes pneumáticos, anestesia hipotensiva, hemotransfusões e agentes farmacológicos, sendo que estes últimos podem se relacionar a complicações inerentes ao seu uso. ${ }^{5,6}$

Vários estudos usando selantes de fibrina tópicos como Quixil® (Omrix Biopharmaceuticals, New York City, NY, EUA) e Floseal ${ }^{\circledR}$ (Baxter International, Deerfield, IL, EUA) mostraram ser efetivos na redução da perda sanguínea visível, da hemorragia total e das taxas de hemotransfusão em ATJs. ${ }^{7-11}$ No entanto, o uso destes medicamentos pode estar relacionado a complicações tromboembólicas e alérgicas devido à presença de agentes estabilizadores de coágulos presentes em suas fórmulas, como ácido tranexâmico (AT) e a aprotinina. ${ }^{12}$ Uma nova geração de selantes de fibrina (Evicel ${ }^{\circledR}$, Johnson \& Johnson, New Brunswick, NJ, EUA), não contendo tais agentes, foi então desenvolvida. ${ }^{13}$

O objetivo do presente estudo foi avaliar o uso intraoperatório de um selante de fibrina humano tópico livre de estabilizadores de coágulo na ATJ quanto à perda sanguínea, à necessidade transfusional, ao tempo de internação hospitalar, à percepção da dor, à amplitude de movimento (ADM)e à incidência de complicações.

\section{Métodos}

A população do estudo foi composta por 64 pacientes submetidos a ATJ para o tratamento da OA primária do joelho, entre setembro de 2015 e abril de 2017.

Pacientes com > 55 anos de idade, apresentando ADM de pelo menos $90^{\circ}$, sem deformidades angulares significativas e com alterações radiográficas de OA compatíveis com o grau 4 ou 5 Ahlback foram incluídos no estudo, após a assinatura do termo de consentimento informado. Em conformidade com os padrões éticos, o estudo foi aprovado pelo Conselho de Ética Institucional (protocolo número 48370515.4.0000.5273).

Os pacientes que apresentaram algum fator de risco predisponente a um aumento do sangramento durante ou após a cirurgia, como coagulopatias, doença renal crônica, tratamento contínuo com anticoagulantes orais e OA secundária, assim como aqueles cujo procedimento cirúrgico durou $>2$ horas foram excluídos.

O tamanho da amostra foi determinado por estudos similares na literatura ${ }^{9}$ e a alocação em dois grupos experimentais, com 32 pacientes cada, foi realizada por randomização usando envelopes selados. Os envelopes foram abertos apenas imediatamente antes do fechamento da ferida, para evitar algum tipo de viés. Os pacientes do grupo de intervenção (Selante) receberam aplicação tópica de $6 \mathrm{~mL}$ do agente hemostático de fibrina Evicel ${ }^{\circledR}$, composto de 
fibrinogênio humano (de 250 a $450 \mathrm{mg}$ ) e trombina (40006000 UI) que vêm em dois frascos separados e são combinados através de um dispositivo aplicador. Os pacientes do grupo de controle (Controle) não receberam nenhuma aplicação do produto.

Todos os pacientes selecionados foram submetidos à mesma técnica cirúrgica padrão (ATJ primária unilateral cimentada com estabilização posterior), sob isquemia pneumática, por cirurgiões com $>5$ anos de experiência. Foram utilizados três modelos de implantes: Press Fit Condylar Sigma DePuy-Synthes ${ }^{\circledR}$ (Johnson \& Johnson, New Brunswick, NJ, EUA), NexGen Knee Replacement System ${ }^{\circledR}$ (Zimmer Biomet, Warsaw, IN, EUA) e ACS Knee System ${ }^{\circledR}$ (Implantcast, Hamburg, Alemanha).

O manguito pneumático foi mantido inflado até o completo fechamento da ferida, evitando a perda sanguínea durante todo o tempo cirúrgico. Todos os pacientes receberam um único dreno Hemovac ${ }^{\circledR}$ (Zimmer Biomet, Warsaw, IN, EUA) de 4,8 mm intra-articular com sucção negativa, mantido durante 24 horas. A prevenção de eventos tromboembólicos foi realizada em todos os pacientes, que receberam uma única dose diária de $40 \mathrm{mg}$ de heparina subcutânea de baixo peso molecular, iniciada de 12 a 24 horas após o término do procedimento e mantida por 15 dias.

Os índices hematimétricos foram medidos pré-operatoriamente, no $1^{\circ}$ dia pós-operatório ( 24 horas, $\mathrm{n}=63$ ) e no $3^{\circ}$ dia pós-operatório ( 60 a 72 horas, $\mathrm{n}=41$ ) para aqueles que ainda não haviam recebido alta até este momento.

Os seguintes parâmetros foram considerados para a análise da perda sanguínea: volume sanguíneo, perda sanguínea no dreno, perda de hemoglobina, e perda sanguínea total.

O volume sanguíneo de cada paciente foi determinado de acordo com a fórmula de Nadler. ${ }^{14} \mathrm{O}$ volume de perda sanguínea drenada foi determinado pelo volume de sangue $(\mathrm{mL})$ coletado por sucção negativa através do dreno de Hemovac ${ }^{\circledR}$, dentro de 24 horas após o procedimento cirúrgico.

A perda de hemoglobina (Hb) após 24 horas foi calculada utilizando a fórmula:

$\mathrm{Hb}$ perdida $=[\mathrm{Hb}$ admissão $-\mathrm{Hb}$ alta $(24$ horas $)]+\mathrm{Hb}$ transfundida

Onde:
(1) $\mathrm{Hb}$ perdida = perda total de $\mathrm{Hb}$ (g/dL) após o procedimento

(2) $\mathrm{Hb}$ admissão $=\mathrm{Hb}(\mathrm{g} / \mathrm{dL}) \times$ Volemia $/ 100$

(3) $\mathrm{Hb}$ alta $=\mathrm{Hb}(\mathrm{g} / \mathrm{dL}) \times$ Volemia $/ 100$

$\mathrm{Hb}$ transfundida = quantidade total de $\mathrm{Hb}(\mathrm{g} / \mathrm{dL})$ transfundida através de concentrados de glóbulos vermelhos. Cada bolsa de sangue do banco de sangue da instituição e do estado contém uma concentração média de $\mathrm{Hb}$ de $60 \mathrm{~g} /$ embalagem $(0,6 \mathrm{~g} / \mathrm{dL})$.

A perda de $\mathrm{Hb}$ após 60 horas foi calculada com base na fórmula:

$\mathrm{Hb}$ perdida $=[$ Hb admissão $-\mathrm{Hb}$ alta $(60$ horas $)]+\mathrm{Hb}$ transfundida

A perda sanguínea $(\mathrm{mL})$ no primeiro dia pós-operatório (24 horas) foi calculada pela seguinte fórmula:

Perda sanguínea $=$ Volemia $\times$ Hb perdida $(24$ horas $) / \mathrm{Hb}$ admissão

A perda sanguínea $(\mathrm{mL})$ no $3^{\circ}$ dia pós-operatório (60 horas) foi calculada pela seguinte fórmula:

Perda sanguínea $=$ Volemia $\times$ Hb perdida $(60$ horas $) / \mathrm{Hb}$ admissão

Coletamos dados sobre todos os pacientes que foram hemotransfundidos e o número de bolsas de hemocomponentes que cada um recebeu.

O tempo total de internação hospitalar foi analisado e comparado entre os grupos. Os critérios de alta foram o controle da dor com analgésicos orais e a capacidade de deambular com auxilio, além da ausência de complicações clínicas.

A intensidade da dor foi avaliada em todos os pacientes através da escala visual analógica (EVA), variando de 1 a 10 . A informação foi coletada no hospital, antes da cirurgia, repetida no $2^{\circ}$ dia e na $6^{\mathrm{a}}$ semana após o procedimento cirúrgico.

A ADM foi avaliada com o paciente em posição supina usando um goniômetro milimetrado padrão.

Buscamos identificar a ocorrência de complicações como problemas de cicatrização de feridas, trombose venosa profunda (TVP) e infecção superficial ou profunda.

Todos os dados foram analisados pelo GraphPad Prism 5 para Windows (GraphPad Software, Los Angeles, CA, EUA). Os resultados foram apresentados com o desvio padrão (DP) médio correspondente. $O$ teste exato de Fisher foi usado para

Tabela 1 Comparativo de gênero, idade, peso, altura, índice de massa corporal e volemia entre os grupos

\begin{tabular}{|l|l|l|l|}
\hline & $\begin{array}{l}\text { Grupo Controle } \\
\boldsymbol{n}=\mathbf{3 1}\end{array}$ & $\begin{array}{l}\text { Grupo Selante } \\
\boldsymbol{n}=32\end{array}$ & valor-p \\
\hline Gênero & 5 masculino e 26 feminino & 4 masculino e 28 feminino & 0,76 \\
\hline Idade (anos) & $69,48 \pm 1,27$ & $69,19 \pm 1,21$ & 0,86 \\
\hline Peso (Kg) & $81,71 \pm 2,0$ & $78,43 \pm 1,69$ & 0,21 \\
\hline Altura (m) & $1,60 \pm 0,01$ & $1,58 \pm 0,01$ & 0,47 \\
\hline IMC & $31,81 \pm 0,73$ & $31,23 \pm 0,72$ & 0,57 \\
\hline Volemia (mL) & $4.450 \pm 110$ & $4.270 \pm 80$ & 0,10 \\
\hline
\end{tabular}

Abreviação: IMC, índice de massa corporal. Valores indicam média \pm desvio padrão. 


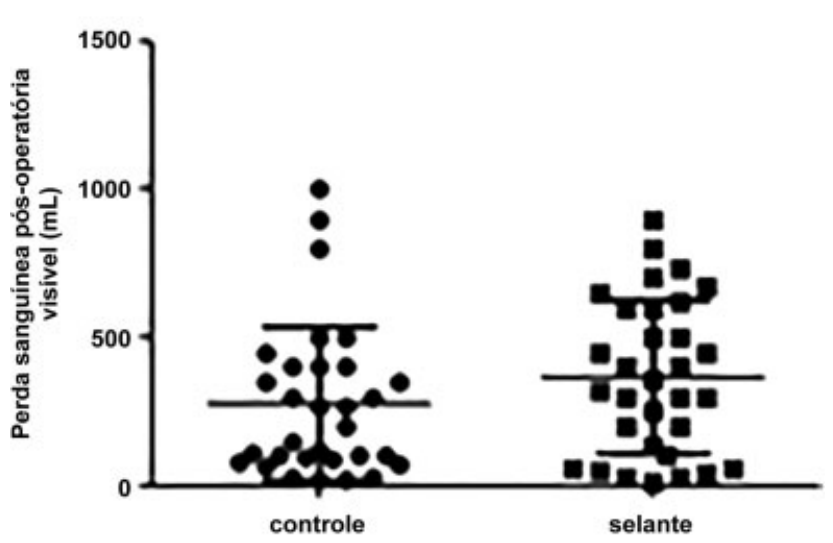

Fig. 1 Perda sanguínea, quantificada no dreno após 24 horas. Realizado teste $\mathrm{t}$ de Student não pareado.

comparar a proporção de gênero entre os grupos. Idade, peso, altura, índice de massa corporal (IMC), volume sanguíneo, perda sanguínea drenada após 24 horas, perda de Hb após 24 horas e 60 horas, perda sanguínea após 24 horas e 60 horas e tempo de internação foram analisados pelo teste $t$ de Student não pareado. A dor (EVA) e a ADM foram analisadas pela análise de variância (ANOVA) de duas vias seguida por um teste $t$ de Student pareado post hoc de Benferroni. Para todas as análises, um valor de $p<0,05$ foi considerado estatisticamente significativo.

\section{Resultados}

Dos 64 pacientes eleitos para o estudo, 1 paciente do grupo controle foi excluído da avaliação de resultados, pois o procedimento cirúrgico ultrapassou as 2 horas antes da conclusão do fechamento total da ferida. Desta forma, 32 pacientes receberam o agente hemostático e 31 pacientes não o receberam. Os grupos de tratamento e controle foram semelhantes em termos de características básicas como gênero, idade, peso, altura, IMC e volemia (-Tabela 1).

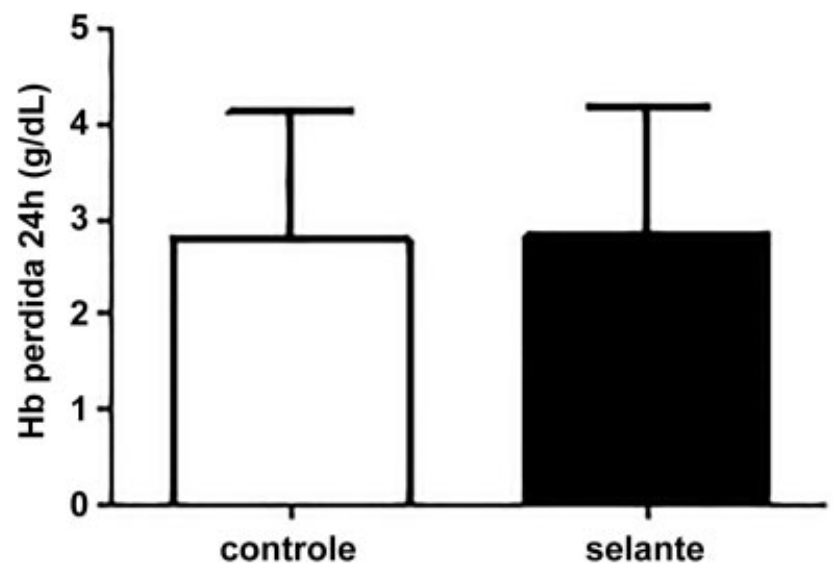

Fig. 2 Perda média de hemoglobina 24 horas após a cirurgia, onde $\mathrm{Hb}$ perdida $=[\mathrm{Hb}$ admissão $-\mathrm{Hb}$ alta $(24 \mathrm{~h})]+\mathrm{Hb}$ transfundida. Realizado teste $t$ de Student não pareado.

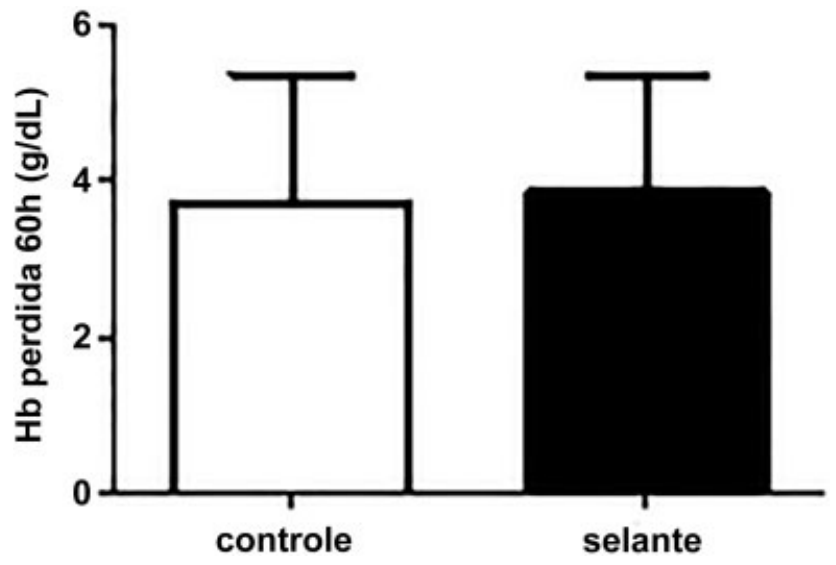

Fig. 3 Perda média de hemoglobina 60 horas após a cirurgia, onde $\mathrm{Hb}$ perdida $=[\mathrm{Hb}$ admissão $-\mathrm{Hb}$ alta $(60 \mathrm{~h})]+\mathrm{Hb}$ transfundida. Realizado teste $\mathrm{t}$ de Student não pareado.

Em relação ao volume de sangramento aferido no dreno em 24 horas, não houve diferença significativa entre os grupos (Controle 276,5 $\mathrm{mL} \pm 46,24$ versus Selante 365,9 $\mathrm{mL} \pm 45,73 ; n=63 ; p=0,17$ ) (-Fig. 1).

Não houve diferença significativa entre os grupos em relação à perda de $\mathrm{Hb}$ média em 24 horas (Controle 2,78 g/dL $\pm 0,24$ versus Selante $2,85 \mathrm{~g} / \mathrm{dL} \pm 0,24 ; n=63 ; p=0,41$ ) (-Fig. 2 ).

Não houve diferença significativa entre os grupos em relação à perda de $\mathrm{Hb}$ média em 60 horas, (Controle 3,69 $\mathrm{g} / \mathrm{dL} \pm 0,39$ versus Selante $3,85 \mathrm{~g} / \mathrm{dL} \pm 0,33 ; \mathrm{n}=41 ;$ $\mathrm{p}=0,37$ ) (-Fig. 3).

Não houve diferença significativa entre os grupos em relação à perda sanguínea total média em 24 horas (Controle $930 \mathrm{~mL} \pm 78$ versus Selante $890 \mathrm{~mL} \pm 67 ; n=63 ; p=0,35$ )

\section{(-Fig. 4)}

Não houve diferença significativa entre os grupos em relação à perda sanguínea total média em 60 horas (Controle $1.250 \mathrm{~mL} \pm 120$ versus Selante $1.190 \mathrm{~mL} \pm 96 ; n=41$; $p=0,34)$ (-Fig. 5).

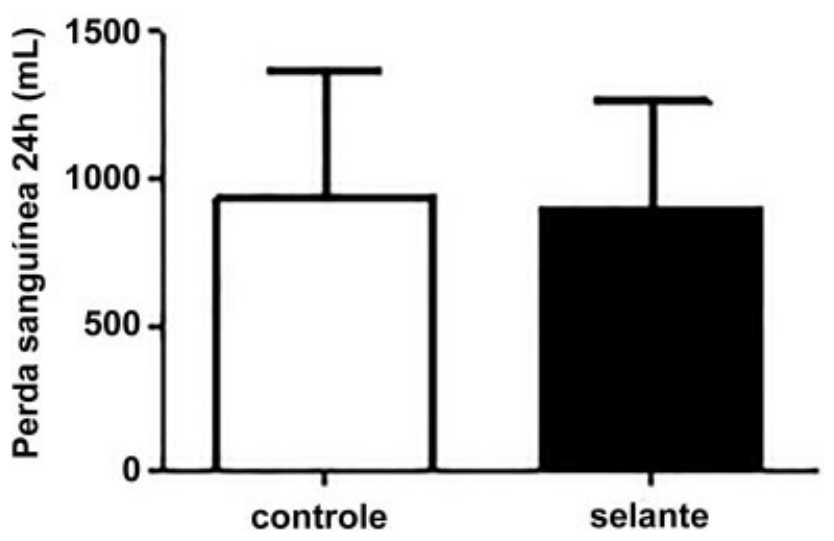

Fig. 4 Perda sanguínea média 24 horas após a cirurgia, onde Perda sanguínea $=$ Volemia $\times$ Hb perdida (24h)/Hb admissão. Realizado teste $\mathrm{t}$ de Student não pareado. 


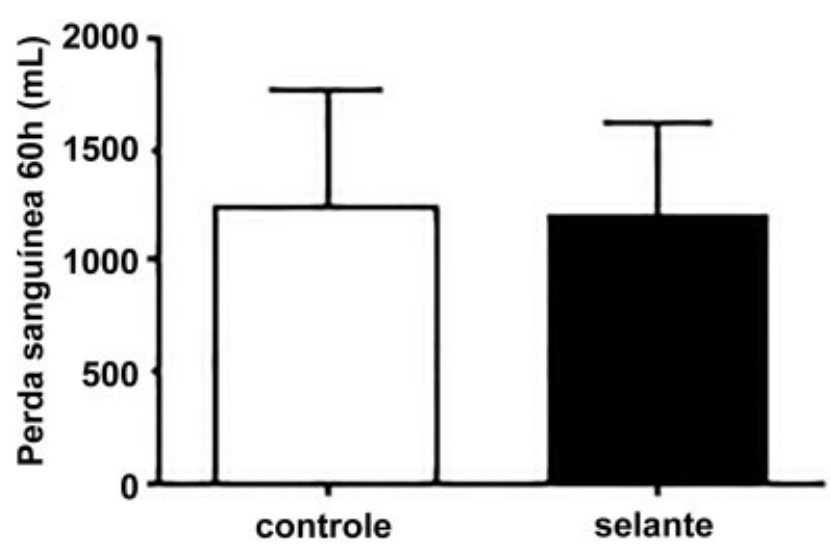

Fig. 5 Perda sanguínea média 60 horas após a cirurgia, onde Perda sanguínea $=$ Volemia $\times \mathrm{Hb}$ perdida $(60 \mathrm{~h}) / \mathrm{Hb}$ admissão. Realizado teste t de Student não pareado.

Apenas um paciente do grupo controle foi transfundido com uma bolsa de concentrado de hemácias. Nenhum paciente do grupo Selante foi transfundido.

Não houve diferença significativa entre os grupos em relação ao tempo de dias de internação hospitalar (Controle $5,61 \pm 0,50 ; n=31$ versus Selante $4,81 \pm 0,36 ; n=32$; $p=0,10)$ (-Fig. 6).

Em relação à EVA, apesar da dor ter reduzido em ambos os grupos ao longo do tempo, não houve diferença significativa entre os grupos $(p>0,05)$ (-Tabela 2 e -Fig. 7)

Em relação à ADM média ao longo do tempo, detalhada na - Tabela 3, esta foi maior no grupo Selante do que no grupo controle no $2^{\circ}$ dia pós-operatório $(p=0,01)$, porém sem diferença significativa na $6^{\underline{a}}$ semana $(p=0,13)$ ( - Fig. 8)

Não houve complicações de cicatrização de ferida, de TVP ou de infecções em nenhum dos grupos.

\section{Discussão}

Em relação ao volume de sangramento aferido no dreno em 24 horas, observamos um sangramento maior no grupo de estudo em relação ao grupo controle, porém sem significância estatística. Skovgaard et $\mathrm{al}^{15}{ }^{15}$ em estudo prospectivo,

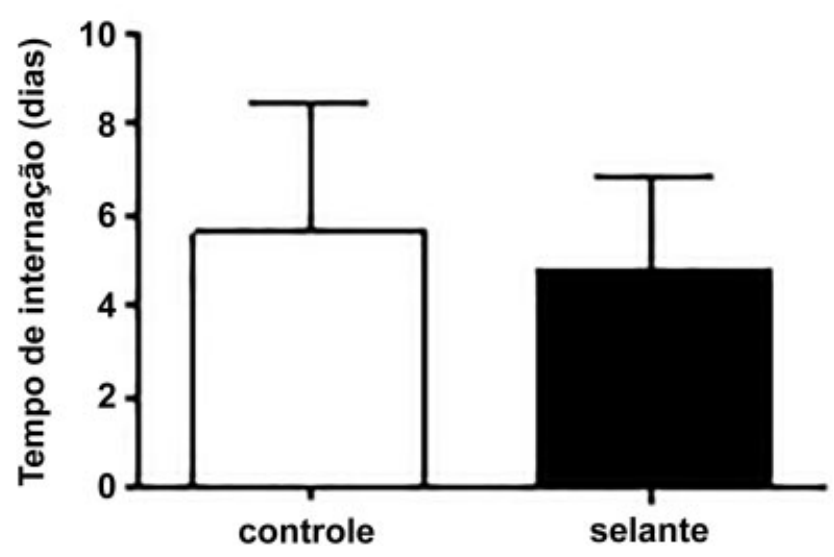

Fig. 6 Tempo de internação, do dia da cirurgia até a alta hospitalar. Realizado teste $\mathrm{t}$ de Student não pareado. duplo-cego, placebo-controlado, avaliando 24 pacientes (48 joelhos) submetidos a ATJ bilateral simultânea, onde um joelho recebeu a aplicação tópica de $10 \mathrm{~mL}$ do selante de fibrina Evicel ${ }^{\circledR}$ e o outro solução salina, não observaram diferenças significativas em relação ao volume de sangue perdido no dreno de 24 horas. Heyse et al $^{16}$ avaliaram 200 pacientes, em um estudo prospectivo e randomizado duplocego em ATJ, no qual 100 pacientes receberam a aplicação de $10 \mathrm{~mL}$ de Evicel® tópico e 100 pacientes nenhuma intervenção adicional. A perda sanguínea no dreno após 24 horas foi significativamente maior no grupo de estudo (780 versus $673 \mathrm{~mL}, p=0,029$ ). Esta perda sanguínea maior no grupo de estudo poderia ser explicada por causa de um "efeito rebote", gerando um maior sangramento após a degradação deste coágulo artificial pela plasmina endógena.

Não observamos diferença significativa entre os grupos em relação à perda de $\mathrm{Hb}$ média nas análises 24 e 60 horas após a cirurgia. Maheshawari et al, ${ }^{17}$ avaliando retrospectivamente a eficácia do selante de fibrina Evicel ${ }^{\circledR}$ (113 pacientes) em relação a um grupo controle (70 pacientes), também não observaram diferenças significativas em relação aos níveis de $\mathrm{Hb}$ em cada um dos 3 primeiros dias de pós-operatório.

Pudemos determinar que não houve diferença significativa entre a perda sanguínea total entre os grupos. Resultados semelhantes foram observados por Randelli et al. ${ }^{4}$ Estes conduziram um estudo para verificar se, em comparação com o controle, a aplicação tópica do novo selante de fibrina (Evicel®) em pacientes submetidos a ATJ primária reduziria a perda sanguínea peroperatória. Neste, 62 pacientes foram randomizados para receber a aplicação tópica de Evicel ${ }^{\circledR}$ ( $n=31)$ ou não $(n=31)$. A perda sanguínea peroperatória foi similar entre os grupos ( $1.900 \mathrm{~mL}$ no grupo controle e $1.800 \mathrm{~mL}$ no grupo de tratamento; $p=0,4)$. Da mesma forma, Heyse et $\mathrm{al}^{16}$ também não encontraram diferenças em relação à perda sanguínea total entre os seus grupos (1.409 mL no grupo controle versus $1.441 \mathrm{~mL}$ no grupo de intervenção; $p=0,44$ )

Reinhardt et al ${ }^{13}$ observaram que a perda sanguínea total foi semelhante, em uma coorte retrospectiva de 114 pacientes submetidos a ATJ, na qual um grupo recebeu o selante de fibrina tópico (Evicel ${ }^{\circledR}$ ) e outro uma infiltração local contendo epinefrina. Considerando que a injeção de adrenalina periarticular local reduz as taxas de sangramento após a ATJ, podemos sugerir que o uso do selante de fibrina foi efetivo. $\mathrm{O}$ autor destaca que, por ser retrospectivo e sem randomização, pode ter havido um viés de seleção.

Um paciente do grupo controle e nenhum do grupo de intervenção foi hemotransfundido em nosso estudo. Randelli et $\mathrm{al}^{4}$ e Maheshwari et $\mathrm{al}^{17}$ encontraram taxas semelhantes de hemotransfusão nos grupos de intervenção e controle, sugerindo que o uso do selante de fibrina não tem efeito sobre as taxas de hemotransfusões.

Bou Monsef et $\mathrm{al}^{3}$ avaliaram retrospectivamente 176 pacientes submetidos a ATJ, comparando o efeito do "cell saver”, do selante de fibrina (Evicel ${ }^{\circledR} 5 \mathrm{~mL}$ ), da doação préoperatória autóloga, e de nenhuma intervenção sobre o sangramento perioperatório e da necessidade de hemotransfusão. Todas as estratégias resultaram em uma redução 
Tabela 2 Escala visual análoga ao longo do tempo em ambos os grupos

\begin{tabular}{|l|l|l|l|}
\hline & $\begin{array}{l}\text { Grupo } \\
\text { Controle }\end{array}$ & $\begin{array}{l}\text { Grupo } \\
\text { Selante }\end{array}$ & valor-p \\
\hline EVA internação & $6,16 \pm 0,33$ & $6,15 \pm 0,43$ & 0,99 \\
\hline EVA 2 ${ }^{\circ}$ dia & $2,58 \pm 0,18$ & $2,75 \pm 0,30$ & 0,63 \\
\hline EVA 6 semanas & $0,59 \pm 0,14$ & $0,71 \pm 0,13$ & 0,52 \\
\hline
\end{tabular}

Abreviação: EVA, escala análoga visual. Valores indicam média \pm desvio padrão.

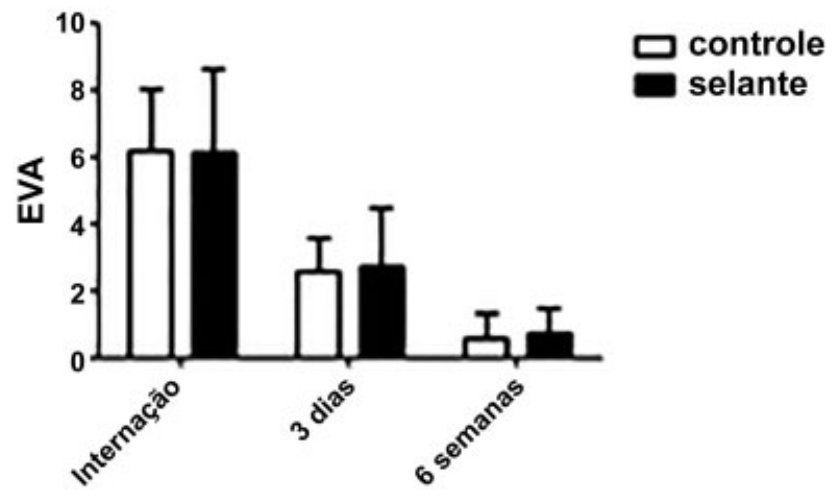

Fig. 7 Escala Visual Análoga médiá dos pacientes em ambos os grupos ao longo do tempo (entre a internação, 2 dias e 6 semanas de pós-operatório). Analisados pela análise de variância (ANOVA) de duas vias seguida por um teste $t$ de Student pareado post hoc.

Tabela 3 Amplitude de movimento ao longo do tempo

\begin{tabular}{|l|l|l|l|}
\hline & $\begin{array}{l}\text { Grupo } \\
\text { Controle }\end{array}$ & $\begin{array}{l}\text { Grupo } \\
\text { Selante }\end{array}$ & valor-p \\
\hline ADM internação & $82,4^{\circ} \pm 19,3^{\circ}$ & $87,8^{\circ} \pm 20,1^{\circ}$ & 0,28 \\
\hline ADM $2^{\circ}$ dia & $76,7^{\circ} \pm 13^{\circ}$ & $84,3^{\circ} \pm 9,7^{\circ}$ & 0,01 \\
\hline ADM 6 semanas & $89,8^{\circ} \pm 8,8^{\circ}$ & $92,8^{\circ} \pm 6,3^{\circ}$ & 0,13 \\
\hline
\end{tabular}

Abreviação: ADM, amplitude de movimento. Valores indicam média \pm desvio padrão.

significativa na necessidade de transfusão de sangue alogênico sobre o controle, sugerindo que o uso de Evicel $₫$ foi uma medida efetiva quando comparado aos demais.

Em relação ao tempo de internação, não houve nenhuma diferença significativa entre os grupos. No estudo de Randelli et $\mathrm{al}^{4}{ }^{4}$ também não houve diferença significativa entre os grupos.

Analisando a dor pós-operatória pela EVA e a ADM ao longo do tempo, não identificamos nenhuma diferença significativa entre os grupos no nosso estudo, exceto quanto à ADM no $2^{\circ}$ dia de pós-operatório, que se mostrou maior no grupo estudo, porém não se manteve na avaliação com 6 semanas. Não conseguimos elaborar uma justificativa para esta diferença. Reinhardt et $\mathrm{al}^{13}$ não observaram diferença significativa entre a ADM após 6 semanas de pós-operatório. Skovgaard et $\mathrm{al},{ }^{15}$ em relação à recuperação funcional, ao inchaço, à dor, à força da extensão do joelho e à ADM após 21

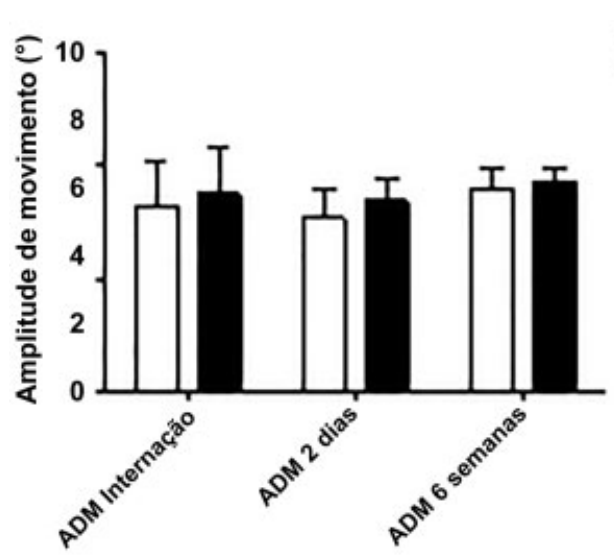

Fig. 8 Amplitude de movimento média dos pacientes avaliados ao longo do tempo, aferido através de goniômetro milimetrado. Analise pela análise de variância (ANOVA) de duas vias seguida por um teste $t$ de Student pareado post hoc.

dias de cirurgia, também não observaram diferença significativa entre os seus grupos. Heyse et $\mathrm{al}^{16}$ também não observaram diferenças em relação ao ganho de ADM e EVA entre os grupos de intervenção e controle.

Nenhum estudo na literatura relatou complicações de cicatrização de feridas, infecciosas ou tromboembólicas relacionadas ao uso do Evicel®, assim como nós também não tivemos nenhuma destas complicações.

Destacamos como pontos fortes o desenho de nosso estudo, prospectivo, randomizado, controlado e unicêntrico, além de termos critérios de inclusão muito bem determinados, o que permitiu que os grupos de tratamento e controle fossem muito semelhantes em termos de características básicas como gênero, idade, peso, altura, IMC e volemia.

Como limitação do nosso estudo, podemos apontar a perda de pacientes na avaliação do $3^{\circ}$ dia, entre 60 e 72 horas de pós-operatório. Julgamos que não seria prudente manter internados pacientes em condições de alta apenas para termos estes dados.

\section{Conclusões}

Concluímos que o agente hemostático de fibrina humano livre de agentes estabilizadores de coágulo não foi eficaz em reduzir o volume de sangramento ou a necessidade de hemotransfusão em ATJ, e que seu uso não foi capaz de interferir positiva ou negativamente sobre o tempo de internação hospitalar, percepção de dor e amplitude de movimento. Seu uso não se relacionou a nenhuma complicação.

Conflitos de Interesses

Os autores declaram não haver conflitos de interesses.

\section{Referências}

1 Lotke PA, Faralli VJ, Orenstein EM, Ecker ML. Blood loss after total knee replacement. Effects of tourniquet release and continuous passive motion. J Bone Joint Surg Am 1991;73(07):1037-1040 
2 Sehat KR, Evans RL, Newman JH. Hidden blood loss following hip and knee arthroplasty. Correct management of blood loss should take hidden loss into account. J Bone Joint Surg Br 2004;86(04): 561-565

3 Bou Monsef J, Buckup J, Waldstein W, Cornell C, Boettner F. Fibrin sealants or cell saver eliminate the need for autologous blood donation in anemic patients undergoing primary total knee arthroplasty. Arch Orthop Trauma Surg 2014;134(01):53-58

4 Randelli F, D’Anchise R, Ragone V, Serrao L, Cabitza P, Randelli P. Is the newest fibrin sealant an effective strategy to reduce blood loss after total knee arthroplasty? A randomized controlled study. J Arthroplasty 2014;29(08):1516-1520

5 Wang H, Shan L, Zeng H, Sun M, Hua Y, Cai Z. Is fibrin sealant effective and safe in total knee arthroplasty? A meta-analysis of randomized trials. J Orthop Surg Res 2014;9(01):36-44

6 Yang TQ, Geng XL, Ding MC, Yang MX, Zhang Q. The efficacy of fibrin sealant in knee surgery: A meta-analysis. Orthop Traumatol Surg Res 2015;101(03):331-339

7 Levy O, Martinowitz U, Oran A, Tauber C, Horoszowski H. The use of fibrin tissue adhesive to reduce blood loss and the need for blood transfusion after total knee arthroplasty. A prospective, randomized, multicenter study. J Bone Joint Surg Am 1999;81 (11):1580-1588

8 Molloy DO, Archbold HA, Ogonda L, McConway J, Wilson RK, Beverland DE. Comparison of topical fibrin spray and tranexamic acid on blood loss after total knee replacement: a prospective, randomised controlled trial. J Bone Joint Surg Br 2007;89(03): 306-309

9 McConnell JS, Shewale S, Munro NA, Shah K, Deakin AH, Kinninmonth AW. Reducing blood loss in primary knee arthroplasty: a prospective randomised controlled trial of tranexamic acid and fibrin spray. Knee 2012;19(04):295-298

10 Sabatini L, Trecci A, Imarisio D, Uslenghi MD, Bianco G, Scagnelli R. Fibrin tissue adhesive reduces postoperative blood loss in total knee arthroplasty. J Orthop Traumatol 2012;13(03):145-151

11 Kim HJ, Fraser MR, Kahn B, Lyman S, Figgie MP. The efficacy of a thrombin-based hemostatic agent in unilateral total knee arthroplasty: a randomized controlled trial. J Bone Joint Surg Am 2012; 94(13):1160-1165

12 Dhillon S. Fibrin sealant (evicel ${ }^{\circledR}$ [quixil ${ }^{\circledR} /$ crosseal $\left.^{\mathrm{TM}}\right]$ ): a review of its use as supportive treatment for haemostasis in surgery. Drugs 2011;71(14):1893-1915

13 Reinhardt KR, Osoria H, Nam D, Alexiades MA, Figgie MP, Su EP. Reducing blood loss after total knee replacement: a fibrin solution. Bone Joint J 2013;95-B(11, Suppl A):135-139

14 Nadler SB, Hidalgo JH, Bloch T. Prediction of blood volume in normal human adults. Surgery 1962;51(02):224-232

15 Skovgaard C, Holm B, Troelsen A, Lunn TH, Gaarn-Larsen L, Kehlet $\mathrm{H}$, et al. No effect of fibrin sealant on drain output or functional recovery following simultaneous bilateral total knee arthroplasty: a randomized, double-blind, placebo-controlled study. Acta Orthop 2013;84(02):153-158

16 Heyse TJ, Haas SB, Drinkwater D, Lyman S, Kim HJ, Kahn BA, et al. Intraarticular fibrinogen does not reduce blood loss in TKA: a randomized clinical trial. Clin Orthop Relat Res 2014;472(01): 272-276

17 Maheshwari AV, Korshunov Y, Naziri Q Pivec R, Mont MA, Rasquinha VJ. No additional benefit with use of a fibrin sealant to decrease peri-operative blood loss during primary total knee arthroplasty. J Arthroplasty 2014;29(11):2109-2112 